$B N-47310$

DE92 015848

\title{
HLNC CALIBRATION AND APPLICATION TO WASTE MEASUREMENT
}

\author{
Ming-Shih Lu \& Theodor Teichmann \\ Brookhaven National Laboratory
}

Philippe-Marie De Ridder \& Calvin Delegand

International Atomic Energy Agency

March 4, 1992

Department of Nuclear Energy

Brookhaven National Laboratory

Associated Universities, Inc.

Upton, Long Island, New York 11973

Under Contract No. DE-AC02-76CH00016 with the

UNITED STATES DEPARTMENT OF ENERGY

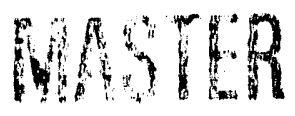

DISIMAIBUTION OF THIS DOCUMENT IS UNLIMTIIED 


\section{DISCL.AIMER}

This report was prepared as an account of work sponsored by an agency of the United States Government. Neither the United States Government nor any agency thereof, nor any of their employees, not any of their contractors, subcontractors, or their employees, makes any warranty, express or implied, or assumes any legal liability or responsibility for the accuracy, completeness, or usefuiness of any information, apparatus, product, or process disclosed, or represents that its use would not infringe privately owried rights. Reference herein io any specific commercial product, process, or service by trade name, trademark, manufacturer, or otherwise, does not necessarily constitute or imply its endorsement, recommendation, or favoring by the United States Government or any agency, contractor, or subcontractor thereof. The views and opinions of authors expressed herein do not necessarily state or reflect those of the United States Government or any agency, contractor or subcontractor thereof. 


\title{
HLNC CALIBRATION AND APPLICATION TO WASTE MEASUREMENT
}

\author{
Ming-Shih Lu, Theodor Teichmann \\ Brookhaven National Laboratory, Upton, New York, U. S. A. \\ Philippe-Marie De Ridder, Calvin Delegard \\ International Atomic Energy Agency, Vienna, Austria
}

\section{§1. Introductory Remarks}

This paper addresses problems of calibration and estimation of a parameter, $\rho_{0}$. in high level neutron coincidence counting (HLNC) measurement. The presentation begins with a deductive approach, based on the generally accepted physical equations which are satisfied by the total and coincidence count rates ${ }^{1,2}$. A new approach for the estimation of $\rho_{0}$ via measurement and a new calibration procedure consistent with the physical theory of coincidence counting measurement ${ }^{1,2}$ are presented. The approach is compared with the existing conventional approach in which possible inconsistency with the theoretical model is shown.

In addition, the original model for neutron coincidence counting in a multiplying medium was extended to simultaneously account for the possible presence of poison, as well as for the neutron detection. ${ }^{3}$ The equations suitable for the data analysis of measurement of waste and their criteria are given.

\section{§2. Iheoretical Considerations}

The basic equations underlying the use of HLNC for the determination of the plutonium content of samples are ${ }^{1,2}$

$1 K$. Bobnel, "The Effect of Multiplication un the Quantitative Determination of Spontaneously Fissioning Isotopes by Neutron Correlation Analysis", Nuclear Science and Engineerina 20. (1985), pp.75-82.

2 W. Hage and D. M. Cifarelli, "On the Factorial Moments of the Neutron Multiplicity Distribution of Fission Cascades", Nuclear Instruments and Methods in Ehvsics Besearch Section A A 236, (1985)pp.165177.

3 M-S. Lu and T. Teichmann, "A Generalized Model for Coincidence Counting", to appear in Nuclear instuments and Mathods in Physics Basaarch. Section A 


$$
\begin{aligned}
& T=\varepsilon M v_{1 s}(1+\alpha) C m \\
& R=\frac{1}{2} \varepsilon^{2} M^{2} f\left[v_{2 s}+\frac{(M-1) v}{v_{1 i}-1} v_{2 i}(1+\alpha)\right] C m
\end{aligned}
$$

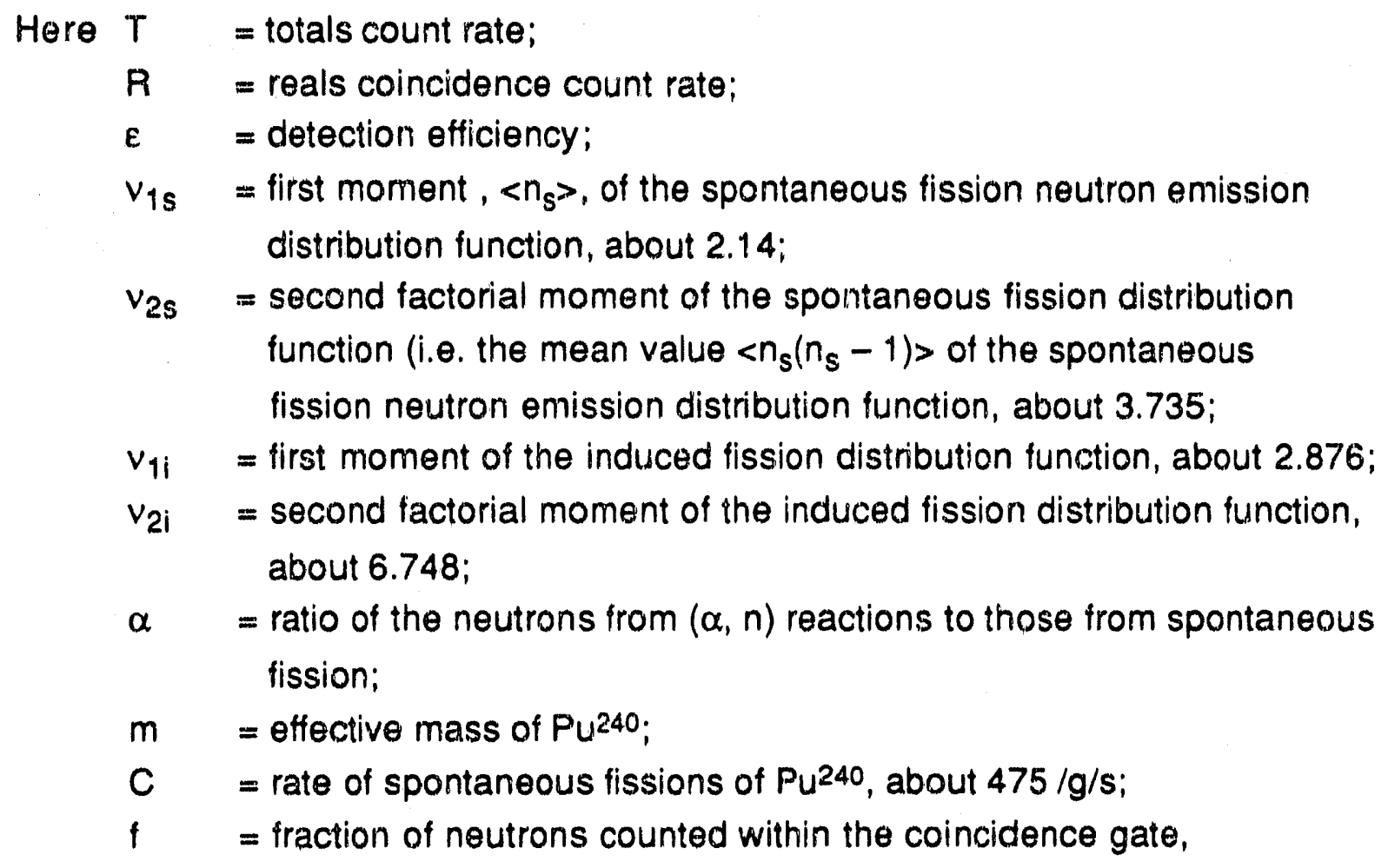

$$
f=e^{-d / \tau}\left(1-e^{-g / \tau}\right)
$$

where $\tau$ is the detector die-away time, $d$ the pre-delay and $g$ the coincidence gate width;

and $M=$ leakage multiplication factor, given by

$$
M=\frac{1-p_{f}}{1-p_{f} v_{1 i}}
$$

where $p_{f}$ is the probability of inducing fission. 
The underlying assumptions in the model are that each neutron in the sample has the same probability to induce a fission and that neutrons with the same pedigree can register a coincidence pair during measurement. The expressions were initially derived independently by Böhnel ${ }^{1}$ and Hage and Cifarelli ${ }^{2}$ considering explicitly only the effect of induced fissions in the sample.

Recently a more physically transparent model was developed. The model accounts simultaneously and explicitly for the effects of induced fissions, poison, as well as instrumental detection. In particular, it was shown that the detection efficiency, $\varepsilon$, in equations (1) and (2) above should be expressed as the product of an intrinsic detector efficiency, $\varepsilon_{0}$, the probability that a neutron impinging on the detector system will register a count in the detector, and the probability that a neutron generated in the sample will reach the detector. In terms of the probabilities of each neutron to induce a fission, $p_{f}$ and the capture probability, $p_{c}$, i.e. the probability of being absorbed (for example, by a poison) without inducing a fission, the detection system efficiency can then be expressed as

$$
\varepsilon=\varepsilon_{0} \frac{1-p_{f}-p_{c}}{1-p_{f}} \text {. }
$$

\section{§3. A New Calibration Precedure}

From equations (1) and (2), calibration is viewed as a procedure to determine the detector efficiency, $\varepsilon$, and the fraction of neutrons counted within the coincidence gate-width, $f$. The properties of the material used in calibrations are assumed known. The calibrated parameters are then used to assay the plutonium content of unknown samples.

Firstly, the detector die-away time for each sample is determined by measuring the coincidence count rates by varying the gate-widths. For example, when the coincidence count rates corresponding to gate-widths of $g_{1}$ and $g_{2}$, where $g_{2}=2 g_{1}$. are $R_{1}$ and $R_{2}$, then

$$
\tau=-g_{1} / \ln \left(R_{2} / R_{1}-1\right)
$$


With this value of $\tau$ and the known pre-delay $d$ and gate-width $g$, the fraction of neutrons counted within the coincidence gate is determined for each sample. (Approximate values for the HLNCII are: $\tau=43 \mu \mathrm{s}, d=4.5 \mu \mathrm{s}, g=64 \mu \mathrm{s}$ and thus $f=0.697$.)

Secondly, the detection efficiency for each sample in the detector during calibration is determined. This is achieved by eliminating $E M$ from equation (1) and then solving equation (2) for $M$, which yields

$$
M=\frac{2 R v_{1 s}^{2}(1+\alpha)^{2} C m-[1-k(1+\alpha)] T^{2}{ }^{2} v_{2 s}}{k(1+\alpha) T^{2}{ }^{2} v_{2 s}} .
$$

Here $k$ is a physical constant of the system

$$
k=\frac{v_{1 s} v_{2 i}}{\left(v_{1 i}-1\right) v_{2 s}}
$$

which occurs both in equation (2) above and in the quadratic equation determining $M$ which is used in a variant of the procedure (see equation (12) below).

The detection efficiency is then obtained from

$$
\varepsilon=\frac{T}{v_{1 s}(1+\alpha) C m M}
$$

With $\varepsilon$ and $f$ for each sample so determined, a statistical computational procedure is then used to obtain an optimum set of $\varepsilon^{*}$ and $f^{*}$ for subsequent applications. If the material to be assayed is similar to the calibration standards, these parameters should not change much between calibration and applications. Various criteria selected by the user, for example, minimum relative variances, minimum absolute total differences (or other criteria) may be used in this statistical process. The statistical variation of $\varepsilon$ and $f$ during the calibration process provide an estimate of the accuracy and precision to be expected during applications. 
The application of the $\varepsilon^{*}$ and $f^{*}$ obtained to determine the plutonium mass is trivial. Similar to conventional HLNC data analysis, the value of $\alpha$ for each sample is assumed known. Equations (1) and (2) are then used to solve for the two unknowns, $M$ and $\mathrm{m}$.

\section{§4. The Conventional Procedure}

The conventional algorithm for HLNC data analysis is obtained by first multiplying equation (2) by $(1+\alpha)$ and then dividing it by equation (1) to get a quadratic equation in $\mathrm{M}$ :

$$
\frac{R(1+\alpha)}{T}=\frac{1}{2} \varepsilon f M \frac{v_{2 s}+\frac{(M-1) v_{1 s}}{v_{1 i}-1} v_{2 i}(1+\alpha)}{v_{1 s}} .
$$

Noticing that the resulting expression still contains the unknown factors $\varepsilon$ and $f$ on the right-hand side, Ensslin ${ }^{4}$ suggests bypassing this problem by introducing the quantity po

$$
\rho_{0}=\left[\frac{R(1+\alpha)}{T}\right]_{0}
$$

where the subscript " 0 " denotes a non-multiplying sample, for which $M=1$. The Ensslin equation for $M$ is then obtained by dividing equation (10) by $\rho_{0}$ to arrive at:

$$
k(1+\alpha) M^{2}+[1-k(1+\alpha)] M-r=0
$$

where $k$ is a physical constant of the system, defined above (see equation (8)), and

4 N. Ensslin. "A Simple Self-Multiplication Correction for In-Plant Use", Proceedinos of the 7th Annual ESARDA Sympesium en Safeouards and Nuclear Materials Management, (Liege, Belgium, 21-23 May, 1985), ESABDA 19, (European Safeguards Research and Development Association, Brussels, Belgium, 1985) pp.233-238. 


$$
r=\frac{R(1+\alpha)}{\rho_{0}^{\top}}
$$

$m$, the effective plutonium-240 mass, is then defined by

$$
R_{c}=\frac{R}{M r}=\frac{\rho_{0}^{\top}}{M(1+\alpha)}=\rho_{0} \varepsilon v v_{1 s} C m \equiv a_{0} m
$$

This last equation defines the conventional calibration constant, $a_{0}$, for the HLNC. Notice that from equations (1) and (2) the quantity $\rho_{0}$

$$
\rho_{0}=\left[\frac{R(1+\alpha)}{T}\right]_{0}=\frac{1}{2} \varepsilon f \frac{v^{2 s}}{v_{1 s}}=\rho_{0}(\varepsilon, f)=\rho_{0}(\varepsilon f)
$$

is a function of $\varepsilon$ and $f$. In order to minimize the changes to the existing conventional algorithm for the calculation of $M$ when the new procedure is used, the quantity $p_{0}$ can be calculated for the measurement system at the calibrated condition, $\varepsilon^{*}$ and $f^{*}$ according to this equation, though its introduction and application are not essential.

If the value $\rho_{0}$ at the measurement condition (i.e., $\varepsilon$ and $f$ ) can be determined, the material properties $M$ and $m$ which are independent of the detector parameters $\varepsilon$ and $f$ can also be determined. The new calibration procedure described in the previous section aims to achieve this by using calibration standards with material properties similar to the materials to be assayed, and thus assumes that the $\varepsilon$ and $f$ so determined will not vary greatly in the further applications.

In the standard approach ${ }^{4}, \rho_{0}$ is presumed to be obtained by measurement of $R$ and $T$ for small masses $(10-20 \mathrm{~g})$, or via measurements of a series of samples of the material with decreasing masses and eventual extrapolation of the ratio $\mathrm{R} / \mathrm{T}$ to zero mass. However, references regarding such experimental determination of $\rho_{0}$ have not been published and the practice is not usually exercised. For example, the zero mass measurement is especially difficult for the Universal Fast Breeder Reactor Subassembly Counter (UFBC) or other fuel assembly coincidence counters. Such measurements may prove particularly difficult due to the much lower signal-to-noise ratio for small samples, as well as the much greater statistical fluctuation of the $\varepsilon$ and $f$ 
values for such samples relative to the larger masses encountered in practice. The $\varepsilon$ and $f$ obtained for small masses may thus deviate greatly from those for larger mass systems of interest.

In fact, a Monte-Carlo simulation on a mainframe computer is usually performed to obtain the value of $\rho$. For HLNCII, the value 0.103 was obtained, and this is frequently used in measurements with HLNCII. However, Menlove 5 has noted that in order to match $M$ between experiment and Monte-Carlo simulation, a value of 0.108 works better. Hybrid methods, combining Monte Carlo techniques with other aspects of the conventional models have also been applied, yielding values of $\rho_{0}$ which vary, sometimes substantially, from $0.103 . .^{5}$ It also turns out that the values so obtained may deviate greatly from that obtained in real applications, leading to undesirable inconsistencies.

In the usual measurement procedure for HLNCII, a constant value of $\rho_{0}=0.103$ is used, regardless of the calibration parameter $a_{0}$ actually measured. From equations (14) and (15) one sees that

$$
a_{0}=\frac{1}{2} \varepsilon^{2}+C v_{2 s}=\frac{2 C v_{1 s}^{2}}{f v_{2 s}} \rho_{0}^{2}
$$

Thus, deviation of $a_{0}$ from its nominal value shows that $\varepsilon$ or $f$ (or equivalently $\rho_{0}$ ) also so deviate, and this should be noted in the further application of the results.

\section{§5. Discussion}

In the current phenomenological approach $a_{0}$ is determined using the fixed (assumed) value of $p_{0}$ by calculating $M$ from equation (12), using the definition (13) of

5 H. O. Menlove, "Calibration and Error Reduction in Neutron Coincidence Counting", Nuc Mater. Manage XVl, \#1 (1987) pp.20-28.

5 J. E. Stewart, "A Hybrid Monte Carlo/Analytical Model of Neutron Coincidence Counting", Irans Am. Nuc Soc. $53,(1986)$, pp.149-151, and J. E. Stewart, et al., "Calibration Parameters from Monte Carto Simulations of Neutron Coincidence Assay of MOX Fuel Elements: A Substitute for Standards?", Proceeclings of the 11th Annual ESARDA Symoosium on Safequards and Nuclear Materials Management, (Luxembourg, 30 May - I June, 1989), ESARDA 22, (European Safeguards Research and Development Association, Brussels, Belgium, 1989) pp.135 - 141. 
$r$, and finally obtaining $a_{0}$ using equation (14). This procedure yields a satisfactory result for the actual case used, and for other measurements for which the efficiency $\varepsilon$, the gate-width factor $f$, and hence the value of $\rho_{0}$ do not change greatly from their nominal values.

Even if the (real) values of $\rho_{0}$ do vary somewhat, this procedure will probably prove adequate, provided that the leakage multiplication factor, $M$, remains near 1 , the point being that

$$
\begin{gathered}
\frac{d M}{M}=-\left[\frac{r}{r+M^{2} k(1+\alpha)}\right] \frac{d \rho_{0}}{\rho_{0}} \\
\approx-0.3 \frac{d \rho_{0}}{\rho_{0}}
\end{gathered}
$$

using results derived elsewhere ${ }^{6}$, i.e. $M$ is not sensitive to changes on $\rho_{0}$. In addition, equation (14) shows that the calibration parameter is effectively

$$
\frac{\rho_{0}}{a_{0}}
$$

so that errors in $\rho_{0}$ may be compensated for by countervailing errors in $a_{0}$. (However this possibility needs to be verified by the method described above.) On the other hand, if the samples are such that because of their configuration, their constitution and the presence of impurities, the value of $M$ is substantially changed, then it becomes necessary to use the relation (16) and the equations from which it is derived to obtain a consistent pair of values of $\rho_{0}$ and $a_{0}$ to calculate the correct values of the Pu240 effective mass $m$.

More careful study of the physical and mathematical relations cited above shows that there are features which may further mitigate the inconsistencies that may arise in the standard approach. Equation (15) shows that $\rho_{0}$ is a function of the product

6 "Analysis of Error Propagation in Gamma Spectroscopy (HRGS) and High Level Neutron Coincidence (HLNC) Counting as Applied to the Non-Destructive Analysis (NDA) of Plutonium-Bearing Materials" Ming-Shih Lu and Theodor Teichmann, (Preliminary Draft) February 1991. 
$\varepsilon f$, and not of $\varepsilon$ and $f$ separately. If the efficiency, $\varepsilon$, is a decreasing function of the neutron energy, while the gate-width factor, $f$, increases as the neutrons slow down ${ }^{7}$, then their product, and hence $\rho_{0}$, may not vary greatly from case to case. If confirmed, the standard approach will be applicable in a wide variety of circumstances to a reasonable degree of approximation.

\section{\$6. Examples}

In order to examine the application of the new procedure described in $\S 3$ above, the data in Table VI of LA-10815-MS ${ }^{8}$ for Sellafield $\mathrm{PuO}_{2}$ samples obtained with the Euratom/LANL. HLNCII counter were used. Since the detector die-away time, and hence the gate-width fraction $f$ for each sample are not known, a constant value $f=$ 0.678 was assumed. (To be rigorous, this assumption should be checked by measurement of the die-away time for each sample.) Samples \#12, 17, and 19 were excluded as described in the document.

For the remaining 16 samples, a calibration constant $a_{0}=18.17$ was obtained when $\rho_{0}=0.103$ was used in the conventional calibration procedure. It is to be noted that near the end of the same report, a new value of $a_{0}=18.02$ was cited based on additional $\mathrm{Cf}$ source measurements to account for sample-container effects and source normalization. This indicates a reduction in efficiency, as can be seen from equation (15). Using the new procedure vaices of $\rho_{0}=0.1026$ and $a_{0}=18.08$ were obtained, and the lower efficiency is directly indicated in this approach. The new procedure thus correctly identified the change in the calibration constants from the original data, without the need for the extraneous confirmatory measurements using the $\mathrm{Cf}$ source. The new procedure also identified the three cited outliers easily because their efficiencies varied more than four standard deviations from the average of the remaining 16 samples.

7 Both of these effects are plausible contributors to the underlying instrument behavior.

8 H. O. Menlove et al. "Field Test and Calibration of Neutron Coincidence Counters for High-Mass Plutonium Samples", LA-10815-MS, UC-15, February 1987 (Los Alamos National Laboratory, Los Alamos, New Mexico, U.S. A.). 


\section{§7. Application to Waste}

Interpretation of coincidence measurements for waste are more complicated due to the possible inhomogeneity in the distribution of sources and the presence of neutron absorbers or moderators in the waste. A self-interrogation ${ }^{9}$ method based on empirical correlations between the fissile mass and the ratio of an inferred induced coincidence counting rate over the totals counting rate has been used for the data analysis. Fast coincident counting including triples has also been suggested. ${ }^{10}$ Despite the simplicity of the model for coincidence counting, it can be used to assess validity of various approximations and to gain further insights to the problem.

Together with equation (5) for the detection efficiency, equations (1) and (2) can also be used to analyze measurement data for waste. However, the introduction of the additional unknown capture probability due to poison in the waste renders the applications of coincidence measurements more difficult. Nevertheless, when the samples are similar in character to the calibration standards (in that the detection efficiencies and die-away time do not vary significantly between samples and standards measurements), the calibration procedure presented earlier can be used to determine the detection efficiency. In particular, the values of $\alpha$ are assumed known for the standards, the leakage multiplication factors $M_{s}$ are determined first from equation (7), and then the probabilities to induce fission, $p_{f}$, are calculated from equation (4). The detection efficiency $\varepsilon$ is estimated from equation (9).

In application, as in the case of nominal materials, an estimate of the $(\alpha, n)$ source rates is needed. For waste, neutron sources are mostly likely to be dominated by the americium $(\alpha, n)$ reactions. Fortunately, as can be seen from equations (1) and (2), the leakage multiplication factor $M$ as determined from the measured ratio of $R / T$ is only weakly dependent upon $\alpha$ for large $\alpha$ for which the $\alpha$ dependent terms dominate $T$ and $R$ in these equations. In other words, when $\alpha>>1$ and

9 P. A. Russo, H. O. Menlove, et. al. , "Evaluation of the Neutron Self-Interrogation Approach for Assay of Plutonium in High $\alpha \cdot n$ Materials", Proceedings of the Third International Conference on Facility Qperations - Safequards Intefface, November 27 -December 4, 1987, San Diego, Calitornia (American Nuclear Society, Inc., La Grange Park, Illinois),p.176.

10 J. B. Wachter, E. L. Adams and N. Ensslin, "Prototype Fast Neutron Counter for the Assay of Impure Plutonium", Ibid. p.194. 


$$
\alpha>\frac{v_{2 s}\left(v_{1 i}-1\right)}{(M-1) v_{1 s} v_{2 i}}-1
$$

the leakage multiplication factor can be determined via

$$
\frac{R}{T}=\frac{\operatorname{EfM}(M-1) v_{2 i}}{2\left(v_{1 i}-1\right)}
$$

The errors in $\alpha$ will not affect $M$ significantly. Substiruting this $M$ into equation (1), the error in the measured Pu mass depends linearly on the error in $\alpha$.

On the other hand, when the fissile content in the waste is small so that the effect of multiplication on the real coincidence count rate $R$ is negligible, or when

$$
1+\alpha \ll \frac{v_{2 s}\left(v_{1 i}-1\right)}{(M-1) v_{1 s} v_{2 i}}
$$

the reals counting rate alone (equation (2) with $M$ approximately 1) can be used to determine the $\mathrm{Pu}$ content. This assumes that the detection efficiency does not deviate significantly from calibration conditions. Using the estimated PU mass, the value of $\alpha$ can then be obtained from the totals count rate, equation (1), and checked for consistency.

\section{§8. Conclusions}

A method has been presented which determines and calibrates the HLNC instrumental parameters, such as the detection efficiency, the gate-width coincidence factor, and the measurement constants $\rho_{0}$ and $a_{0}$, for coincidence counters in which the multiplication must be taken into account, based on the measurement of samples with well characterized samples, using the underlying physical equations. This method does not need zero mass measurement, nor the extrapolation of data from small mass measurement, nor does it require a Monte Carlo simulation to match the measurement. When the calibration samples are chosen judiciously, the estimated parameters are more pertinent to the associated inspection situations. 
The method can be implemented easily, and should be tested by more actual measurements. In any case, it removes a possible theoretical inconsistency in the conventional procedure. 
$-a_{0}$

$s$
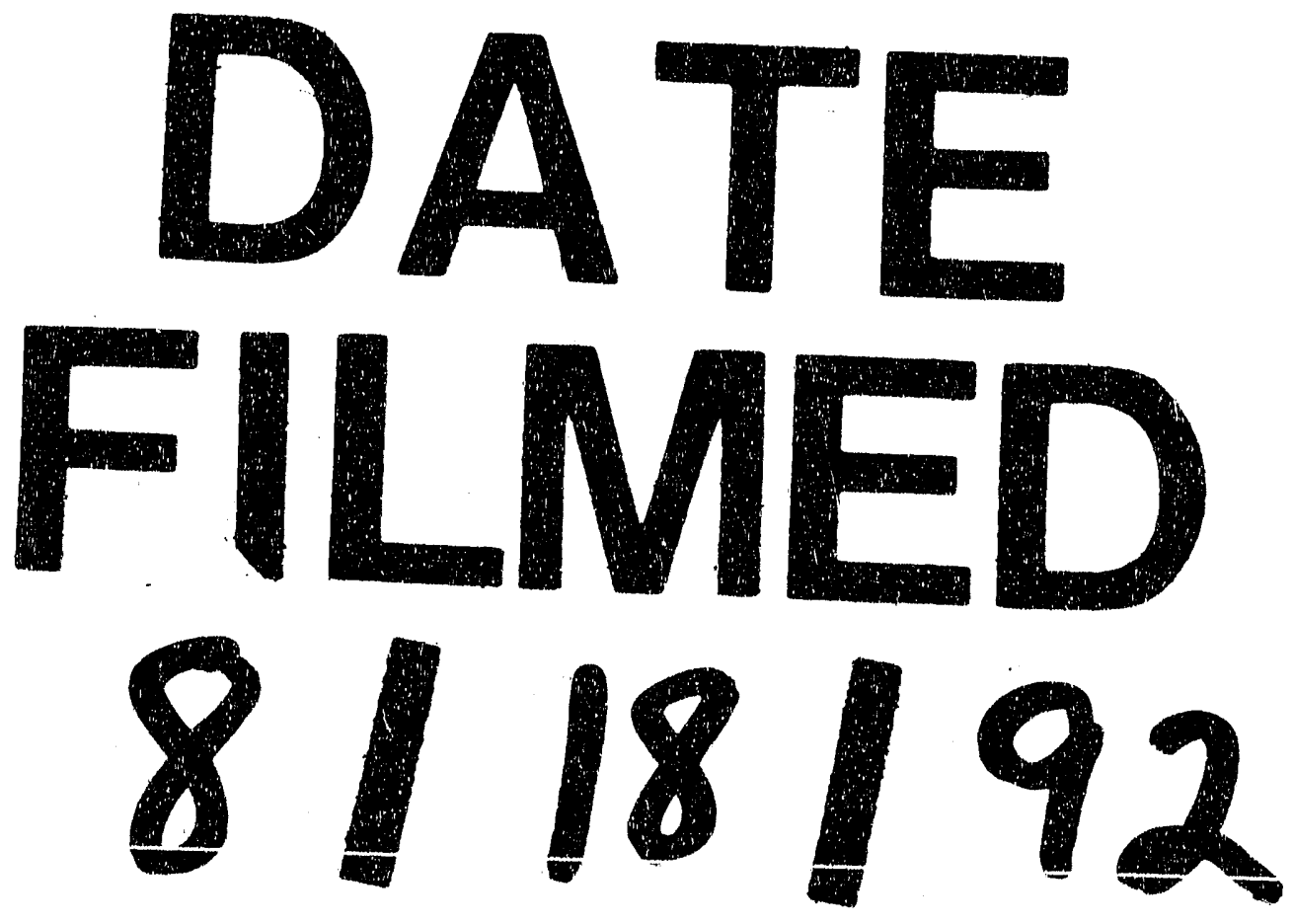
